\title{
Implementing Augmented Reality in the Classroom
}

\author{
Douglas Robert Miller, Tonia Dousay \\ University of Wyoming
}

\begin{abstract}
Augmented reality (AR) is a tool that holds much promise in terms of its application for educational purposes. Despite the fact that the hardware needed to access $A R$ is becoming ubiquitous in classroom environments, however, AR's use in educational settings is diffusing at a slow pace. Several challenges are present for educators interested in adopting AR in their instructional environments but these are not insurmountable. Increasing hardware availability as well as software advances are affording more instructors access to the tools needed to design, develop, and implement $A R$ in and around their classroom. This paper first identifies a conceptual definition of AR that can be easily applied across contexts and then outlines several guidelines for instructors looking to implement AR learning experiences at a classroom level.
\end{abstract}

Keywords: Augmented Reality; Mobile Learning; Constructivism; Social Learning; Best Practices; Classroom Technology

Augmented reality (AR) describes the application of technologies to enhance a physical space or object with relevant information that is presented in a digital medium (Dunleavy, 2014; Klopfer \& Sheldon, 2010). As early adopters of AR have learned more about the tools that allow this type of experience to occur, a more concrete definition of AR has begun to form. AR has been labeled an emerging technology (Dunleavy, 2014) with the implication that there are still barriers that need to be addressed before large-scale adoption will occur. AR is also being defined more broadly, however. Singh and Singh (2013) indicated that current examples of AR include mobile navigation applications and location based advertising, which are both being accomplished through available mobile technologies. This broader definition may be taken to mean that adoption of AR is well underway.

The EcoMOBILE project being conducted by researchers at Harvard is an example of a more advanced application of AR in an education specific context (Dede, 2011). During implementation of the EcoMOBILE project, students equipped with Internet-connected mobile devices visit pre-determined locations around a local pond. When students arrive at any of the pre-determined locations, augmented information triggered by an image or a GPS mechanism, is presented to learners on the mobile device. The media presented is designed to enhance students' understanding of an object or occurrence that takes 
place at the specific location where the augmentation is triggered. Learning goals associated with the implementation of EcoMOBILE are science related and focus on improving students' understanding of ecosystems.

AR is possible due to recent technological advances, but as a learning experience, AR represents more than just the merging of hardware, software, and contextually relevant information (Holden, 2014). AR when applied to education is best defined as a concept, rather than any specific combination of technologies (Chang, Lee, Liang, \& Wu, 2013). Defining AR as a concept allows for the use of a broad array of hardware, software, and design strategies (Chang et al., 2013), which can encourage the development of local, contextually relevant AR learning experiences.

It is also important to define AR in a way that allows it to be modified and adapted to fit local instructional and curricular needs (Mitchell, 2011). When considering AR in terms of its adoption as an innovation, Rogers (2003) indicates that perceived compatibility positively affects the rate of adoption. A conceptual definition of AR is inherently more compatible, because it affords curricular flexibility as well as hardware and software flexibility for instructors who wish to design/develop AR at the classroom level. Holden (2014) presents the case for locally constructed AR, stating:

By learning how to grow and sustain AR game development for use within individual communities, we may be able to reach more than enthusiasts and institutions, and greatly increase the capacity for the successful adoption of further mobile-based pedagogical interventions." (pp. 42-43).

AR has not seen widespread diffusion at the classroom level in recent years (Dede, Dunleavy, \& Mitchell, 2009). It is clear, however, that the rate of mobile technology diffusion across classrooms is increasing (Annetta, Burton, Cheng, Chmiel, \& Frazier, 2012). The increase in hardware availability at a classroom level, along with a broadened definition of what AR actually is, positions the concept of AR as an accessible approach for instructors to use in and around their classrooms. This paper will seek to identify guidelines for implementing a conceptual definition of AR at the classroom level.

\section{Pedagogical Alignment}

There are a number of pedagogical attributes that make AR an ideal instructional tool for a variety of subject areas. AR is immersive and can be linked with the theory of situated learning as well as constructivist methods of instruction (Chang et al., 2013; Dunleavy, 2014; Wasko, 2013). When information is presented using AR in a contextually relevant environment it can enable a greater understanding of how new information is practically applied in realistic settings (Dede, 2012). Contextual learning increases the relevance of new information for students and allows them to see more clearly how new knowledge can impact their environment (Dikkers, Gagnon, Martin, \& Squires, 2014). 
AR can provide support for learners as they need it, especially when AR is available on students' mobile devices. This type of support aligns with Bruner's (1977) spiral curriculum theory that states learning can occur at any time so long as support is provided that meets the immediate needs of the learner. AR also connects with Vygotsky's (trans. 1978) theory of the Zone of Proximal Development (ZPD), which argues that people learn best when provided with timely support from a capable mentor. Further, well-designed contextual AR experiences align with social constructivist tenets of teaching and learning that argue knowledge is shaped in part by the environment in which it is derived (Cheng \& Tsai, 2013). Lee (2012) summarizes these attributes well stating “. . AR has the potential to further engage and motivate learners in discovering resources and applying them to the real world from a variety of different perspectives that have never been implemented in the real world" (pp. 32, 2012).

\section{Before Implementing AR}

Before introducing a new method of instruction into a learning environment it is important for instructors to consider why they want to do so (Smith \& Ragan, 2005). Instructors should note that AR has shown more promise as a supplemental method of instruction rather than as a primary method (Billinghurt \& Dunser, 2012). Research also indicates that the novelty of AR as a method of instruction will not remain after learners become accustomed to it (Dede et al., 2009). This strengthens the argument for using AR as a supplemental (as opposed to primary) method of instruction and also highlights the need for purposefully designed AR implementations. Research further demonstrates AR is most effective when learners possess a certain level of technological expertise, as well as a degree of autonomy typically found with learners at the middle school or high school level (Cheng \& Tsai, 2013). It is therefore not recommended AR be used to facilitate learning in grade levels where instructors will be required to spend time providing technical and cognitive support at levels that render the learning activity ineffective. This is largely a subjective measure, but previous studies have found that when instructors spend any time providing technical support, it detracts from the activity's ability to help learners reach curricular goals (Mitchell, 2011). Recommendations point to a need for learners to be able to assist one another with navigating the AR experience as well as a need for instructors to be very familiar with the AR hardware and software before it is implemented for educational purposes (Mitchell, 2011).

\section{Goal Alignment}

One advantage AR possesses that should be leveraged when developing a learning experience is allowing learners to "see the unseen" (Dunleavy, 2014 p. 28; Wang \& Yoon, 2014, p. 54). Capitalizing on this unique affordance begins with determining which learning goals require students to imagine themselves in situations they would typically be unable to experience.

The power in AR, which sets it apart from traditional curricula and even from purely virtual learning environments, lies in truly augmenting the physical landscape using digital technologies to enable students to see the world around 
them in new ways and engage with realistic issues in a context with which the students are already connected. (Klopfer \& Sheldon, 2010, p. 86)

Considering the potential AR has to connect learners with new knowledge in contextually relevant environments, the variety of subjects and related learning goals around which instructors can compose AR experiences is sizeable. Goals related to environmental science, political science, physics, mathematics, or history in which a learner must imagine an occurrence that typically happens only in a theoretical sense or an event that simply occurs outside of the classroom are all well suited for the potential application of AR to facilitate learning. A review of AR research by Chang, Lee, Liang, \& Wu (2013) further indicates AR instruction commonly employs role-playing, relies on the interaction of learners with a physical location, and stresses specific learning tasks. Lessons that occur in a classroom setting already integrating those pedagogical approaches are well suited for converting into an AR learning experience.

\section{The AR System}

AR has become increasingly associated with the affordances provided by mobile computing devices. One of the affordances that is often used to trigger augmented media is a device's location recognition capability, most commonly based on the Global Positioning System (GPS) (Dede, 2009). Using GPS to trigger augmented media works best in outdoor environments where satellite signals are less likely to be interrupted. A better method for triggering augmented media indoors involves the use of a device's camera in conjunction with specialized software. The software can match an image taken by the camera with a customized database in order to serve information about the image back to the user. Both of these capabilities help ensure the AR experience is contextually relevant and are now available on almost all consumer level smartphones and tablets (Churchill \& de Sa, 2012). It is important that the AR system used takes advantage of the ubiquitous capabilities of modern mobile devices like Internet connectivity, GPS functionality, and/or image capture, so that a broad array of design strategies can be used (Dede, 2012).

\section{AR Hardware}

The hardware used to implement AR at a classroom level is technologically complex but has become increasingly affordable and common in modern learning environments (Singh \& Singh, 2013). Considering the current trend towards ubiquity of smartphone and tablet devices in school systems, it is clear that if AR is implemented by classroom instructors on a broad scale, smartphones and tablets have the most potential to be the devices on which these experiences are developed (Annetta et al., 2012). Nearly all mobile devices are now equipped with GPS functionality and image capture abilities, which allows instructors to select any number of mobile devices to use in delivering an AR experience. Which specific device is used may also depend on decisions made by local school administrators. As long as a device possesses GPS capability or an image-capturing mechanism as well as the ability to connect to a wireless Internet connection, a 
robust AR experience can be designed.

It is important to understand that using mobile devices to construct AR experiences does require some infrastructure. Most notably, AR that relies on the use of mobile devices will require connection to a wireless Internet service, because much if not all of the augmented information that is provided to users will be drawn from data sources available on the Internet. Information can be stored directly on a device for retrieval, but Internet connectivity allows for users to access information in real-time from a seemingly endless number of sources.

If local school policy allows, many students now bring the devices needed to access AR with them to class, making the implementation of AR possible for more instructors in more places (Annetta et al., 2012). This is known in many areas as a Bring Your Own Device (BYOD) initiative. If schools are encouraging learners to bring these devices into the classroom, teachers will need to find creative ways of using them to engage with their learners (Dikkers et al., 2014). AR allows an instructional approach that can take advantage of the increasing presence of mobile devices by applying emergent design strategies to create experiences that aid in the transfer of specific, contextually relevant knowledge to the learner (Dede, 2011). When designing an AR experience for use within a BYOD classroom, the next step is to select AR software that is compatible with the various devices being brought in by students. Even if the devices being used are pre-determined and not a part of a BYOD initiative, it remains important that the selected software is compatible with the hardware.

\section{AR Software}

Just as the hardware that allows AR to be incorporated into learning experiences is becoming more widely available, so too is the software that allows learners to view, and even create, AR experiences (Holden, 2014). Free software packages such as ARIS, Layar, and Junaio allow instructors to implement customized AR experiences (Annetta et al., 2012). Some AR software requires little to no development experience, although other platforms require the designer to possess software development skills. Software selection and the robustness of the AR experience will partially depend on the software development skills of the person who is designing it (Annetta et al., 2012). If the designer of the AR experience does not have software development skills, more common platforms can be used (Billinghurst \& Dunser, 2012). Free platforms such as Yelp (www.yelp. com) have been used to facilitate AR language instruction by directing learners to locate food trucks and discuss them on Yelp in Spanish (Holden, 2014). What is most important when selecting an AR platform is that the platform chosen affords learners the ability to achieve the defined learning goals using research-based pedagogy (Billinghurst \& Dunser, 2012).

It is also important not to ignore the affective front-end experience when designing AR experiences (Cheng \& Tsai, 2013). Cheng and Tsai (2013) indicate that learners' perceptions of AR experiences as they relate to " . . . satisfaction, enjoyment, fun, entertainment, helpfulness, motivation, esthetic pleasure, support of creativity, reward, and emo- 
tional fulfillment" (p. 459) have an impact on the overall AR learning experience. Although ultimately an AR platform should selected that can be managed by both instructor and learners, these affective factors should also be considered when selecting AR software.

\section{Designing AR}

It is important to set realistic media development goals when designing AR learning experiences (Holden, 2014). We have already noted that when selecting hardware and software one should take into account the technological expertise of the individuals who will use it. The same type of thinking should be applied to designing the AR experience. Manageability needs to be evaluated throughout the design and development process.

It is also important for instructors to design AR experiences that are manageable in implementation. Even AR implementations that provided high levels of technical support for instructors presented classroom management issues that affected learning (Dede et al., 2009; Mitchell, 2011). We recommend instructors avoid complex designs that require support beyond the level available during the actual AR implementation. Specific recommendations to address management issues include: training student leaders to support other students during the AR implementation as well as ensuring instructors are familiar with the technologies used before implementing the AR experience (Mitchell, 2011).

\section{Make it Contextual}

One of AR's biggest advantages is its ability to create contextual learning experiences (Dede et al., 2009). The linking of a physical space or object with augmented information helps facilitate ". . . the development of process skills such as critical thinking, problem solving, and communication utilized through interdependent collaborative activities" (Dede et al., 2009, p. 20). Instructors deploying AR should take advantage of this ability. Doing so will ensure the locations used as triggers for AR experiences are provided with new meanings that learners can use to increase their understanding of complex phenomena that occur in the real world (Klopfer \& Sheldon, 2010).

Schools provide diverse environments within which contextual AR experiences can be constructed. What is important is that the subject matter being presented is relevant to the space in which the learner is located (Singh \& Singh, 2013). For example, Annetta et. al. (2012) describe a contextual AR experience that was implemented as an AR game focused on the subject of recycling conducted at multiple sites in and around a single school building. The game was designed to explain how recycling impacts landfill waste while adhering to environmental science teaching standards (Annetta et. al., 2012). The augmented content provided to learners at each location was directly related to the location in which it was presented.

Using contextually appropriate content also adheres to the contiguity principle, which asserts that text or audio should align with the graphics with which it is presented (Clark \& Mayar, 2008, Ch. 5). This principle is easily adaptable for AR, by aligning text or audio 
with appropriate physical spaces or objects.

Instructors can use tools such as Google Maps or an internal map of their own campus to plan out the location of the triggers that activate the augmented information that learners will receive. One best practice is to focus on items that are easily overlooked and use those items as triggers. In general, the AR design should help learners view typically overlooked elements in their environments with a more critical eye (Dunleavy, 2014).

\section{Make it Gamified}

Another positive outcome associated with AR is its ability to motivate students who are not typically engaged by standard methods of instruction (Dede et al., 2009).

By combining gamification elements, such as scoring systems or fail states, to an immersive narrator, the AR designer can leverage two of the most engaging elements of multiple genres (e.g., historical fiction, role playing games) while also reinforcing the learning objectives. (Dunleavy, 2014, p. 30)

Dunleavy (2014) adds that gamified design elements should enable and then challenge learners during the AR experience. Klopfer \& Sheldon (2010) argue for the use of another gamified element in AR by claiming an AR learning environment should afford learners multiple paths to completion. AR experiences that do not challenge learners to ask questions or that allow for simple linear progressions through the material do not necessarily provide egaging learning experiences.(Klopfer \& Sheldon, 2010). In contrast, using gamified design elements helps to ensure an AR learning environment is aligned with situated learning theory as well as constructivist pedagogy (Dunleavy, 2014). We therefore strongly recommend that AR designers incorporate elements of gamification into the learning experiences they create.

\section{Student Driven Design}

Instructors who do not have time to plan new lessons may be interested in allowsing students to drive the creation of the AR experience (Dikkers et al., 2014; Holden, 2014). Student driven AR design helps increase the relevance of the content to learners while enforcing learning goals related to "problem solving, collaboration, critical thinking, and citizenship" (Dikkers et al., 2014, p. 40). This approach is not without its own management challenges and some caution should be taken when deciding to use this method (Dikkers et al., 2014). Employing student driven design does not decrease the importance of defining learner expectations, ensuring all learners have access to the necessary tools, and verifying that all learners possess the pre-requisite knowledge needed to design the AR activity. Student driven AR design has many benefits but the decision to use this method, like all other instructional decisions should be made after determining that the necessary tools, time and support are present to facilitate the process. 


\section{Modifying Designs}

Another approach is to modify existing AR implementations for local purposes. This is possible if the original AR design was developed on an easily customizable platform and addresses a topic that can be applied across contexts. This is the goal of the ECO Mobile project detailed in this paper's introduction (Dede, 2011). The researchers involved are interested in how to enable this type of experience to be implemented on a large scale while maintaining affordability and eliminating other issues that currently prevent widespread diffusion of AR learning experiences. (Dede, 2011). The Time Lab 2100 project developed by researchers at MIT is another example of an AR platform that is designed to be contextual and portable (Klopfer \& Sheldon, 2010). "It was designed to be connected to important real-world locations, but ones that were generic enough for example, a bus stop - that reasonable substitutes could be found in other locations" (Klopfer \& Sheldon, 2010, p. 89).

\section{AR Challenges}

As instructors become more prepared to implement AR there are challenges to be considered. Some of these issues are systemic and instructors will not be able address them individually. Nonetheless, it is important for instructors to be aware of them and bring them to the attention of administrators, technologists and other potential sources of assistance.

Dede (2011) notes that for mobile devices to truly enable the type of ever-present, contextual learning experiences AR can afford, it is important to address several common difficulties. First, a balance must be found between supporting a traditional desktop computing infrastructure and modern wireless computing infrastructure. Schools often struggle to decide where to invest limited funds available for technology infrastructure; instructors who wish to use mobile devices to implement AR must be aware of how these decisions affect their ability to offer this form of instruction. Next, Dede (2011) highlights the need to address issues related to the safety and privacy of students. This is a concern that will be felt across all areas of instruction, as more student information is stored on cloud-based servers. Singh and Singh (2013) also note student privacy as a potential challenge to widespread AR deployments. Dede (2011) also points to a need for incentives to encourage innovation in education-specific AR markets. This is based on the notion that there are not enough reasons for innovators to invest in developing more user-friendly education-specific AR platforms. Although the necessary hardware is becoming less of an issue (Dikkers et al., 2014), more software is needed that would allow instructors to create AR without extensive programming and software development skills (Billinghurst \& Dunser, 2012; Dede et al., 2009).

As is the case with other forms of instruction that use multimedia, instructors must be aware of the potential for AR to create cognitive overload (Chang et al., 2013; Singh \& Singh, 2013). Finally, student safety must remain a top priority. The immersive nature of AR may foster an experience where learners become unaware of their immediate sur- 
roundings, leading to potential physical danger (Chang et al., 2013). One way to ensure the safety of learner is to implement AR in locations without traffic or other hazards that could lead to injury if students become too immersed in the experience.

\section{Conclusion}

It is currently possible for instructors to design, develop, and implement AR learning experiences in their classrooms. Challenges, when they arise, are usually related to varying levels of access to the hardware and software needed to implement AR, though these challenges are waning. The current trend towards a ubiquitous presence of mobile devices in classrooms is positioning $A R$ as an accessible approach for instructors interested in creating immersive, contextual experiences for their learners. The necessary level of technical expertise can present a challenge if creative instructional design is not employed. Leveraging existing devices and software along with the use of creative instructional design will ensure AR is available to more instructors in more classrooms.

This paper sought to provide an increased understanding of how AR can be implemented at a classroom level by first defining AR as a concept. Defining AR conceptually helps broaden access to AR by eliminating the need for specific hardware or software that may or may not be available to all instructors. Further, this paper identified guidelines to consider when designing and developing AR to help ensure it is pedagogically sound and manageable during implementation. Ongoing examinations of AR should investigate how instructors can continue applying existing tools and low to no cost software in ways that address specific learning objectives. Continuing to refine and share how AR is applied in education will facilitate more successful AR experiences designed and developed by instructors at a classroom level. 


\section{References}

Annetta, L., Burton, E. P., Cheng, R., Chmiel, M., \& Frazier, W. (2012). Augmented reality games: Using technology on a budget. Science Scope, 36(3), 54-60.

Billinghurst, M., \& Dunser, A. (2012). Augmented reality in the classroom. Computer, 45(7), 56-63.

Bruner, J. S. (1977). The process of education. Cambridge, Massachusetts: Harvard University Press.

Chang, H., Lee, S. W., Liang, J. C., \& Wu, H. K. (2013). Current status, opportunities and challenges of augmented reality in education. Computers \& Education. 62, 41-49. doi: 10.1016/j.compedu.2012.10.024

Cheng, K. H. \& Tsai, C. C. (2013). Affordances of augmented reality in science learning: Suggestions for future research. Journal of Science Education and Technology, 22(4), 449-462. doi: 10.1007/s10956-012-9405-9

Churchill, E. F., \& de Sa, M. (2012, September). Mobile augmented reality: Exploring design and prototyping techniques. Paper presented at the meeting of HumanComputer Interaction with Mobile Devices and Service, San Francisco, CA.

Clark, R. C., \& Mayer, R. (2008). E-learning and the science of instruction: Proven guidelines for designers of multimedia learning $\left(3^{\text {rd }}\right.$ Ed.). San Francisco, CA: Wiley \& Sons.

Dede, C. (2012). Customization in immersive learning environments. In Dede, C. \& Richards, J (Eds.), Digital teaching platforms: Customizing classroom learning for each student (119-133). New York, NY: Teachers College Press.

Dede, C. (2011). Emerging technologies, ubiquitous learning, and educational transformation. In Kloos, C. D., Gillet, D., Crespo García, R. M., Wild, F., \& Wolpers, M. (Eds), Towards ubiquitous learning (pp. 1-8). Heidelberg, Germany: Springer.

Dede, C. (2009). Immersive interfaces for engagement and learning. Science, 323(5910). 66-69. doi: 10.1126/science.1167311

Dede, C., Dunleavy, M., \& Mitchell. (2009). Affordances and limitations of immersive participatory augmented reality simulations for teaching and learning. Journal of Science Education and Technology, 18(1), 1573-1839. doi: 10.1007/s10956-0089119-1

Dikkers, S., Gagnon, D., Martin, J., \& Squire, K. (2014). Participatory scaling through augmented reality learning through local games. TechTrends, 58(1). 35-41. 
Dunleavy, M. (2014). Design principles for augmented reality learning. TechTrends 58(1), 28-34.

Holden, C. (2014). Homegrown augmented reality. TechTrends, 58(1), 42-48.

Klopfer, E. \& Sheldon, J. (2010). Augmenting your own reality: Student authoring of science-based augmented reality games. New Directions for Youth Development, 2010(128), 85-94. doi: 10.1002/yd.378

Lee, Kangdon. (2012). The future of learning and training in augmented reality. InSight: A Journal of Scholarly Teaching, 7, 31-40.

Mitchell, R. (2011). Alien contact!: Exploring teacher implementation of an augmented reality curricular unit. Journal of Computers in Mathematics and Science Teaching, 30(3), 271-302. Retrieved from: http://www.editlib.org/p/36011

Rogers, E. M. (2003). Diffusion of Innovations (5th ed.). New York, NY: Free Press.

Singh, M. \& Singh, M. P. (2013). Augmented reality interfaces. IEEE Internet Computing, 17(6), 66-70.

Smith, P.L., \& Ragan, T.J. (2005). Instructional design ( $3^{\text {rd }}$ ed.). Hoboken, NJ: Wiley \& Sons.

Vygotsky, L. S. (1978). Mind in society: The development of higher psychological processes. M. Cole, V. John-Steiner, S. Scribner, \& E. Souberman (Eds.), Cambridge, Massachusetts: Harvard University Press.

Wang, J. \& Yoon, S. (2014). Making the invisible visible in science museums through augmented reality devices. TechTrends, 58(1), 49-55.

Wasko, Christopher. (2013). What teachers need to know about augmented reality enhanced learning environments. TechTrends, 57(4), 17-21. 